\title{
Traction screws to reduce a bilateral pedicle fracture of L5: a case report
}

\author{
Nikolaus Kögl, MD, MSc, Martin Dostal, MD, Alexander Örley, MD, Claudius Thomé, MD, and \\ Sebastian Hartmann, MD, PhD
}

Department of Neurosurgery, Medical University of Innsbruck, Tyrol, Austria

\begin{abstract}
Pedicle fractures are rare and usually associated with repetitive stress, high-speed trauma, osteoporosis, unilateral spondylolysis, or instrumentation surgery. A review of the current literature on bilateral pedicle fractures of the lumbar spine revealed only a few cases listed as a complication of instrumentation or excessive decompression surgery. The authors present the clinical case of a 49-year-old man with exacerbating low-back pain and intermittent L5 radicular pain. The known comorbidities were rather remarkable for systemic lupus erythematosus and osteopenia. Radiological investigations revealed an acute bilateral pedicle fracture of L5 without any evidence of preexisting spondylolysis. An off-label minimally invasive fracture reduction and fixation was performed using traction screws and intraoperative navigation. The patient reported instant pain relief and did not show any sensorimotor deficits at discharge. The postoperative CT scan revealed an ossification of the former fracture after 3 months, with great 1 -year follow-up outcome. This is the first documented report on the effectiveness of traction screws used in a patient with bilateral pedicle fractures of the L5 vertebra. This minimally invasive technique represents a promising treatment option in selected cases by sparing segmental fusion.
\end{abstract}

https://thejns.org/doi/abs/10.3171/2020.1.SPINE191229

KEYWORDS pedicle fracture; traction screws; minimally invasive surgery; lumbar

1 BILATERAL pedicle fracture of the lumbar spine is a rare disease, with only a few cases described in the literature. Some of these fractures have been observed in athletes as a consequence of repetitive stress, especially in hyperextended alignment, but this type of fracture can also occur in elderly patients due to reduced bone mass density. ${ }^{1-3}$ The pedicle is known for its strength, making it resistant to shear forces; thus, this anatomical structure represents the most feasible anchorage point in spinal instrumentation procedures. ${ }^{4}$

Pedicle fractures predominantly occur as a surgical complication due to adjacent-segment disease or repetitive mechanical stress at the lumbar spine. The L5 vertebral pedicle is especially prone to fracture as a result of narrow and short anatomical conditions accompanied by high mechanical loads. ${ }^{5}$

Patients may present with a preexisting unilateral spondylolysis, making them prone to a contralateral stress fracture. ${ }^{6}$ Affected patients report excruciating pain after lifting heavy objects. In some cases, the patients experience radicular pain due to mobile fracture elements of the affected ipsilateral pedicle chafing the exiting nerve root. MRI reveals a hyperintense signal at the affected pedicle, and $\mathrm{CT}$ depicts the fracture with or without affecting the nerve root caudally. In the absence of neurological deficits and fracture displacement, conservative treatment should be considered. Nonoperative management includes pain medication and, most importantly, lumbosacral immobilization using a brace. ${ }^{7}$ A transforaminal lumbar interbody fusion (TLIF) with transpedicular screw fixation may be the gold standard in pedicle fractures adjacent to instrumented spine levels or in de novo pedicle stress fractures if conservative treatment fails. This procedure entails a segmental spondylodesis with all its potential disadvantages. Minimally invasive fusion might lead to a better outcome due to less intraoperative blood loss and a reduced operative time and length of inpatient stay, but it also carries the same long-term sequelae by affecting the adjacent seg- 

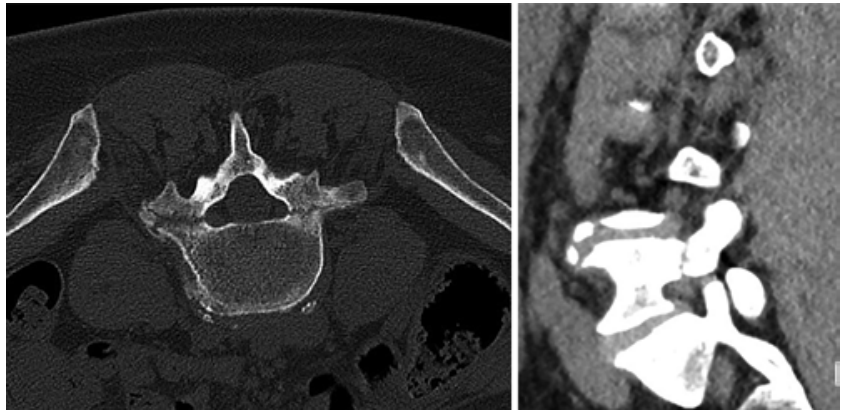

FIG. 1. Preoperative axial (left) and sagittal (right) CT scans of a bilateral fracture of the $L 5$ pedicles.

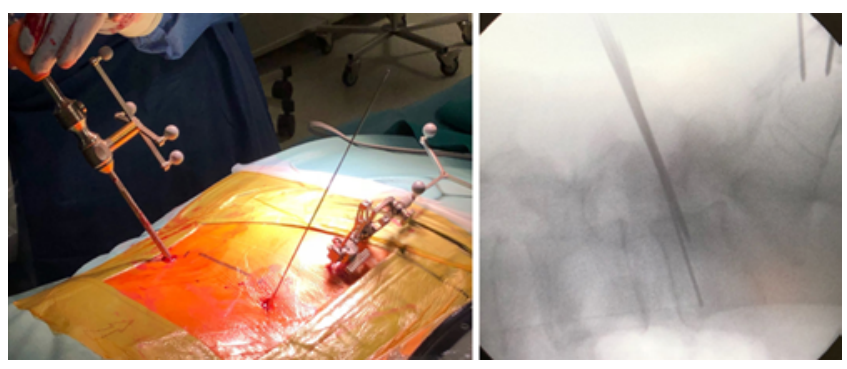

FIG. 2. The patient is placed prone with the reference array attached to the left iliac crest. Left: CT-guided insertion of the awl on the right side, with a K-wire in place on the left side. Right: Awl placement in the pedicle of the L5 vertebra with the help of the navigation system and additional fluoroscopy. Figure is available in color online only.

ment. Simple fixation by using traction screws to reduce the fracture gap has not yet been reported. We report the case of a patient with a bilateral pedicle fracture of L5 treated with minimally invasive implantation of traction screws (CSS, DePuy Synthes) used off label without performing a fusion procedure. These cannulated screws with a 7.3-mm diameter are commonly used in fixation of large fragments such as a femoral head or in case of an iliosacral dislocation.

\section{Case Report}

\section{Presentation and Examination}

A 49-year-old man had experienced acute low-back pain (numeric rating scale score 7 ) for 4 days after lifting a bag of potatoes (approximately $5 \mathrm{~kg}$ ). No further trauma was reported. No neurological deficits were revealed on physical examination. The patient's back pain exacerbated in an upright position as well as in reclination. On mobilization, the patient could provoke right-sided L5 radicular pain with deterioration of the low-back pain.

The patient's past medical history was remarkable for systemic lupus erythematosus and cerebral vasculitis, without permanent neurological deficit. The therapeutic regimen included Endoxan (cyclophosphamide) in the past and corticosteroids, CellCept (mycophenolate mofetil), and Quensyl (hydroxychloroquine) at present.

Corticosteroids had caused a femoral head necrosis in 2011, which was treated with a right-sided total hip replacement. A fall in 2014 led to a fracture of the superior pubic ramus, and a stress fracture of the left fifth metatarsal was reported. Osteopenia was diagnosed using dualenergy x-ray absorptiometry with a hip T-score of -1.9 and a lumbar spine T-score of -1.5. A CT scan (Fig. 1) revealed a bilateral pedicle fracture of L5, which was confirmed by various MRI sequences showing a hyperintense signal at the L5 pedicle bilaterally. A possible compression of the L5 nerve route by the moving fragments was assessed using flexion-extension radiographs.

Surgical management was discussed with the patient, and he gave informed consent prior to surgery after conservative treatment had failed. A minimally invasive navigated pedicle stabilization of the L5 pedicle bilaterally was planned with the off-label use of traction screws.

\section{Surgical Procedure}

The procedure was performed with the patient prone and under general anesthesia, with the reference array for intraoperative navigation attached to the iliac crest. An intraoperative low-dose CT scan (iCT, SOMATOM Definition AS, Siemens Healthcare $\mathrm{GmbH}$ ) was obtained. The Spine \& Trauma 3D Navigation software with the corresponding navigation system (Brainlab AG) was used for image guidance. A percutaneous technique (Viper 2, DePuy Synthes) via a Wiltse approach was chosen for minimally invasive screw placement (Fig. 2). The awl was then placed in the pedicle of the L5 vertebra with the help of the navigation system, and K-wires were inserted through the awl, followed by iCT scanning (Fig. 3). The probes confirmed a good intrapedicular position, so that
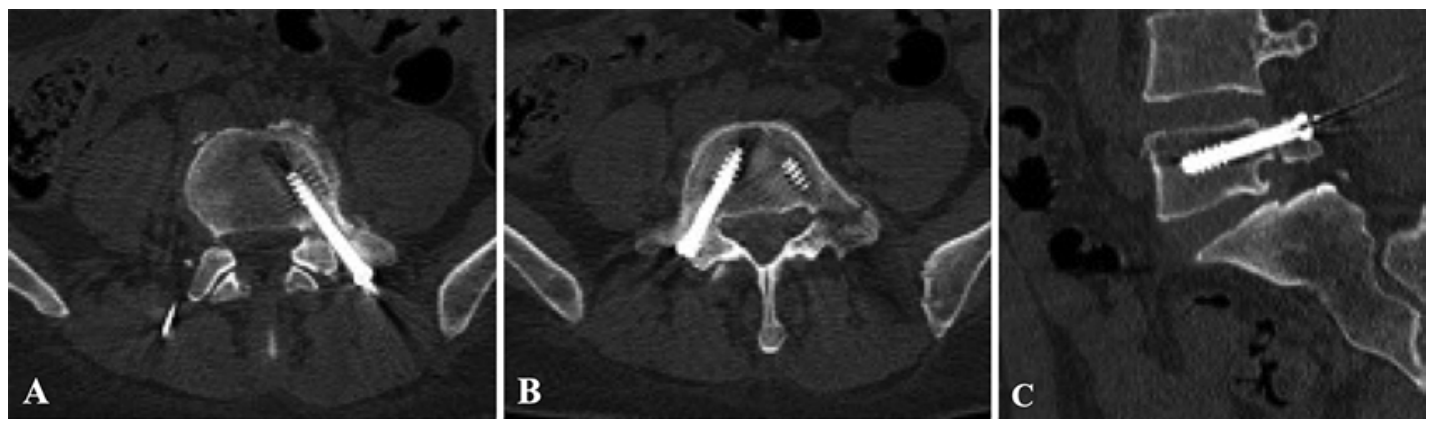

FIG. 3. Intraoperative axial (A and B) and sagittal (C) CT scans obtained after screw insertion. 


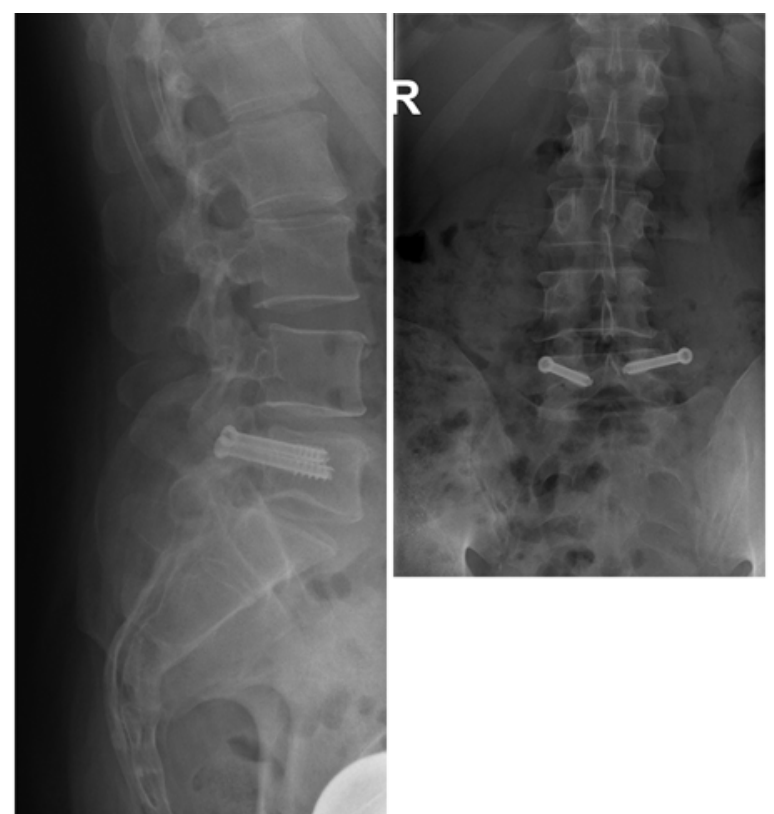

FIG. 4. Postoperative lateral (left) and anteroposterior (right) plain radiographs showing good implant positioning.

tapping with a lead tap of $6.5 \mathrm{~mm}$ in diameter according to the placement of the 7.3-mm tension screw (CSS, DePuy Synthes) under additional fluoroscopic control was accomplished. Cannulated traction screws were then implanted with the help of the placed K-wires to reduce the fracture. A CT scan confirmed sufficient reduction of the fracture on the right side. The left tension screw was further tightened before removing the K-wire (Fig. 4). The procedure lasted approximately 40 minutes.

\section{Postoperative Course}

Pain relief was rapidly achieved, and the patient's lowback pain improved to numeric rating scale score 2 immediately after surgery. Radiography prior to discharge demonstrated adequate reduction of the fracture (Fig. 4).

There was no neurological deficit or any residual pain after 3 months postoperatively. A postoperative CT scan revealed an ossification of the former fracture (Fig. 5) without any signs of screw loosening or failure after 1 year.

\section{Discussion}

Bilateral pedicle fractures are rare. These fractures might occur due to severe trauma, conditions with repetitive stress, osteoporosis, tumors, or unilateral spondylolysis. ${ }^{3,7-9}$ A pedicle fracture can also be seen as a postoperative complication after fusion as a sign of adjacent-segment overloading, especially in patients with osteoporosis..$^{10,11}$

In the case presented, osteopenia was diagnosed based on dual energy x-ray absorptiometry findings of the spine and hip. Osteoporosis has been shown to cause pedicle fractures of the adjacent noninstrumented pedicle in patients who have undergone instrumented spinal fusion cranially and caudally. ${ }^{2}$ In most described cases, fusion surgery was proposed either based on preoperative instability or because of preexisting adjacent spondylodesis. ${ }^{1-3}$

In cases of nondisplaced pedicle fractures and in the absence of neurological deficits, conservative treatment has been advocated. Pedicle fractures tend to heal spontaneously with external bracing. However, nonoperative treatment is associated with long-term immobilization and the potential risk of pseudarthrosis..$^{12}$ Instability or spondylolisthesis due to pedicle fractures may cause disc lesions with progressive degeneration, so that instrumented fusion might be a treatment option.,13 After failed conservative treatment, including painkillers and immobilization using a brace, the patient in our report was offered surgery to increase the likelihood of fracture healing and instant pain relief and to allow an earlier return to work. We decided to perform percutaneous minimally invasive CT-guided off-label pedicle instrumentation without interbody fusion to reduce the fracture and spare the adjacent segments. Traction screws were inserted using a navigation system with additional intraoperative fluoroscopy in order to detect fragment dislocation.

Bilateral pedicle fractures mimic spondylolysis with potential instability. The literature provides evidence that several systems have been used to compress the lytic defect, such as screws (Buck, also described by Bonnici et al.), cerclage (Scott), a modified hook screw (Morscher, described by Albassir et al.), screws and plates (Louis), and modified cerclage (Salib et al.). ${ }^{14-19,24}$ None of these procedures were performed in a percutaneous fashion or with the use of intraoperative navigation. The idea was to spare the adjacent segment through a direct and noninvasive repair by compressing the fracture, as is commonly
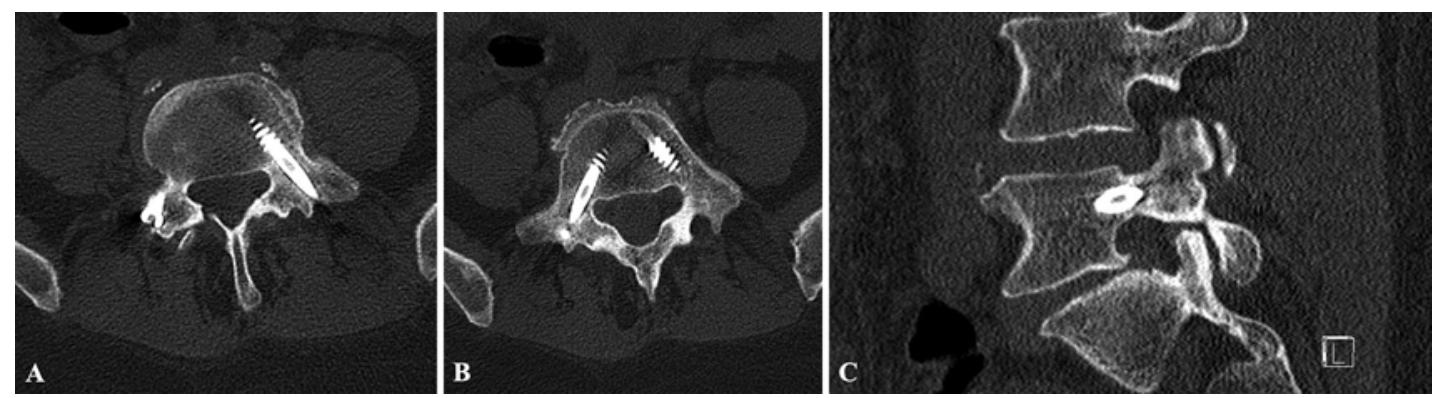

FIG. 5. Postoperative axial (A and B) and sagittal (C) CT scans showing sufficient ossification/fusion of the former bilateral fractures after 3 months postoperatively. 
done in trauma surgery. These minimally invasive procedures have become popular in recent years, reducing blood loss and intraoperative soft-tissue trauma. ${ }^{20}$ Furthermore, postoperative pain and rehabilitation time may be reduced using a minimally invasive TLIF technique compared with open surgery. The challenges of minimally invasive TLIF are represented in an exponential learning curve and the significantly higher radiation exposure compared with conventional open surgery. ${ }^{21}$ Nevertheless, in experienced hands, advantages for the operating staff as well as the patients are reported. ${ }^{22}$ In our case hardly any blood loss was documented, and the operation time was 40 minutes, as a percutaneous technique was chosen. Minimally invasive techniques generally show a faster recovery time, so that patients can return back to work after approximately 7-9 weeks postoperatively. ${ }^{23}$ The patient in the presented case returned to work after 6 weeks.

\section{Conclusions}

This is the very first documented report of the effectiveness of traction screws used in a patient with bilateral pedicle fractures of the L5 vertebra. This meticulous technique was performed with intraoperative low-dose CT scanning and fluoroscopy to detect suboptimal screw positioning and possible fracture dislocation as well as optimal fracture reduction. Bilateral pedicle fractures are rare but may occur in osteopenia. In case of conservative treatment failure, instability, or in the presence of neurological deficits, monosegmental fusion is advocated. However, traction screws may represent a potential minimally invasive alternative by sparing segmental fusion.

\section{References}

1. Karabay N, Ozer E, Ada E. Multi-level, bilateral pedicle fractures: case report. Turk Neurosurg. 2015;25(2):340-343.

2. Kim SW, Kim HS, Kim HS. Unusual osteoporotic stress fracture: adjacent bilateral pedicle fractures. Int J Rheum Dis. 2017;20(5):662-663.

3. Parvataneni HK, Nicholas SJ, McCance SE. Bilateral pedicle stress fractures in a female athlete: case report and review of the literature. Spine (Phila Pa 1976). 2004;29(2):E19-E21.

4. Gunzburg R, Fraser RDM. Stress fracture of the lumbar pedicle. Case reports of "pediculolysis" and review of the literature. Spine (Phila Pa 1976). 1991;16(2):185-189.

5. Knight RQQ, Chan DPKP. Idiopathic scoliosis with unusual stress fracture of the pedicle within solid fusion mass. A case report. Spine (Phila Pa 1976). 1992;17(7):849-851.

6. Sadiq MZ. Bilateral pedicle stress fracture in the lumbar spine of a sedentary office worker. Eur Spine J. 2006;15(suppl 5):653-655.

7. Vialle R, Mary P, de Carvalho A, et al. Acute L5 pedicle fracture and contralateral spondylolysis in a 12-year-old boy: a case report. Eur Spine J. 2007;16(suppl 3):316-317.

8. Luther E, Urakov T, Vanni S. Percutaneous instrumentation of a complex lumbar spine fracture with bilateral pedicle dissociation: case report and technical note. J Neurol Surg A Cent Eur Neurosurg. 2018;79(5):416-423.

9. Wilkinson RH, Hall JE. The sclerotic pedicle: tumor or pseudotumor? Radiology. 1974;111(3):683-688.

10. Ha K-Y, Kim Y-H. Bilateral pedicle stress fracture after instrumented posterolateral lumbar fusion: a case report. Spine (Phila Pa 1976). 2003;28(8):E158-E160.
11. Stanley D, Smith TW. Contralateral pedicle stress fracture. An unusual complication of laminectomy. Spine (Phila Pa 1976). 1990;15(6):598-599.

12. Surur S, Zeglinski-Spinney A, El Koussy MM, et al. Spontaneous atraumatic bilateral pedicle insufficiency fractures. Can J Neurol Sci. 2018;45(6):717-720.

13. Weatherley CR, Mehdian H, Berghe LV. Low back pain with fracture of the pedicle and contralateral spondylolysis. A technique of surgical management. J Bone Joint Surg Br. 1991;73(6):990-993.

14. Albassir A, Samson I, Hendrickx L. Treatment of painful spondylolysis using Morscher's hook [in French]. Acta Orthop Belg. 1990;56(2):489-495.

15. Bonnici AV, Koka SR, Richards DJ. Results of Buck screw fusion in grade I spondylolisthesis. J R Soc Med. 1991;84(5):270-273.

16. Buck JE. Direct repair of the defect in spondylolisthesis. Preliminary report. J Bone Joint Surg Br. 1970;52(3):432-437.

17. Hefti F. Direct repair of spondylolysis with the hook screw [in German]. Orthopade. 1997;26:769-773.

18. Louis R. Fusion of the lumbar and sacral spine by internal fixation with screw plates. Clin Orthop Relat Res. 1986;(203):18-33.

19. Scott J. The Edinburgh repair of isthmic (Group II) spondylolysis. J Bone Joint Surg Br. 1987;69:491-499.

20. Scheufler K-M, Dohmen H, Vougioukas VI. Percutaneous transforaminal lumbar interbody fusion for the treatment of degenerative lumbar instability. Neurosurgery. 2007;60(4) (suppl 2):203-213.

21. Tian W, Xu Y-F, Liu B, et al. Computer-assisted minimally invasive transforaminal lumbar interbody fusion may be better than open surgery for treating degenerative lumbar disease. Clin Spine Surg. 2017;30(6):237-242.

22. Tschugg A, Hartmann S, Lener S, et al. Minimally invasive spine surgery in lumbar spondylodiscitis: a retrospective single-center analysis of 67 cases. Eur Spine J. 2017;26(12):3141-3146.

23. Wang X, Borgman B, Vertuani S, Nilsson J. A systematic literature review of time to return to work and narcotic use after lumbar spinal fusion using minimal invasive and open surgery techniques. BMC Health Serv Res. 2017;17(1):446.

24. Salib RM, Pettine KA. Modified repair of a defect in spondylolysis or minimal spondylolisthesis by pedicle screw, segmental wire fixation, and bone grafting. Spine (Phila $\mathrm{Pa}$ 1976). 1993;18:440-443.

\section{Disclosures}

Dr. Thomé reports the following: consultant for DePuy Synthes, Signus Medical, and Intrinsic Therapeutics; honoraria from Aesculap, DePuy Synthes, Medtronic, Intrinsic Therapeutics, Icotec, NuVasive, Signus Medical, and Zeiss; and non-study-related research support from DePuy Synthes, Medtronic, Intrinsic Therapeutics, Pfizer, Icotec, and Signus Medical.

\section{Author Contributions}

Conception and design: Kögl, Dostal. Acquisition of data: Kögl. Drafting the article: Kögl. Critically revising the article: Kögl, Dostal, Thomé, Hartmann. Reviewed submitted version of manuscript: Kögl, Örley, Thomé, Hartmann. Approved the final version of the manuscript on behalf of all authors: Kögl. Study supervision: Hartmann. Performed surgery: Kögl, Hartmann, Örley.

\section{Correspondence}

Nikolaus Kögl: Medical University of Innsbruck, Tyrol, Austria. nikolaus.koegl@tirol-kliniken.at. 